\title{
A tool for calculation of ${ }^{7} \mathrm{Li}(p, n)^{7} \mathrm{Be}$ neutron source spectra below the three-body break-up reaction threshold
}

\author{
Rebecca Pachuau $^{1}$, B. Lalremruata ${ }^{1,}$,, N. Otuka ${ }^{2}$, L.R. Hlondo ${ }^{1}$, L.R.M. Punte ${ }^{1}$, and H.H. Thanga ${ }^{1}$ \\ 1 Department of Physics, Mizoram University, Tanhril - 796004 Aizawl, Mizoram, India \\ 2 Nuclear Data Section, Division of Physical and Chemical Sciences, Department of Nuclear Sciences and Applications, \\ International Atomic Energy Agency, 1400 Wien, Austria
}

\begin{abstract}
We developed a new deterministic neutron source spectrum code EPEN - Energy of Proton Energy of Neutron - for a given lithium target thickness, sample angular coverage and proton energy from the reaction threshold to the three-body break-up threshold. The angular differential cross sections of the ${ }^{7} \mathrm{Li}\left(\mathrm{p}, \mathrm{n}_{0}\right)^{7} \mathrm{Be}$ and ${ }^{7} \mathrm{Li}\left(\mathrm{p}, \mathrm{n}_{1}\right)^{7} \mathrm{Be}$ reactions evaluated by Liskien and Paulsen were adopted above $1.95 \mathrm{MeV}$ while the functional form suggested by Macklin and Gibbons was adopted for the ${ }^{7} \mathrm{Li}\left(\mathrm{p}, \mathrm{n}_{0}\right)^{7} \mathrm{Be}$ reaction cross section near threshold. The spectra obtained by EPEN are validated by the experimental spectra and also compared with the spectra predicted by two Monte Carlo codes, SimLiT and PINO. The results of comparison are discussed in detail.
\end{abstract}

\section{Introduction}

The ${ }^{7} \mathrm{Li}(\mathrm{p}, \mathrm{n})^{7} \mathrm{Be}$ reaction has been extensively used for experimental studies of neutron-induced reactions because of the rapid rise of the cross section in the nearthreshold region. These low energy neutrons are useful for fundamental studies in radiobiology and dosimetry and also for nuclear astrophysics applications. It is also a candidate of the neutron source reaction for boron neutron capture therapy (BNCT) which is a cancer treatment modality.

For proton energies above $2.37 \mathrm{MeV}$, a second neutron group due to the ${ }^{7} \mathrm{Li}\left(\mathrm{p}, \mathrm{n}_{1}\right)^{7} \mathrm{Be}$ reaction appears at lower energies in addition to the ${ }^{7} \operatorname{Li}\left(\mathrm{p}, \mathrm{n}_{0}\right)^{7} \mathrm{Be}$ neutrons. The ${ }^{7} \mathrm{Li}\left(\mathrm{p}, \mathrm{n}+{ }^{3} \mathrm{He}+\alpha\right)$ three-body break-up channel also opens at proton energies above $3.70 \mathrm{MeV}$ and shows a broad neutron spectrum. The contribution of the low energy background neutrons has to be carefully determined and subtracted especially when one applies the activation technique to measure neutron capture cross sections which decrease as the neutron energy increases in general. However, it is impossible to perform the subtraction based on the neutron source spectra measured by timeof-flight and multiple foil activation techniques at some accelerator facilities. Therefore, experimentalists using such accelerators have to rely upon the calculated neutron source spectra for subtraction of the low energy background neutron contribution.

We have recently checked the ${ }^{7} \mathrm{Li}(\mathrm{p}, \mathrm{n})^{7} \mathrm{Be}$ neutron source spectra predicted by SimLiT [1] and PINO [2] to analyze our neutron activation cross sections measured

\footnotetext{
a e-mail: marema08@gmail.com
}

at the Folded Tandem Ion Accelerator Facility (FOTIA) of the Bhabha Atomic Research Centre (BARC). But the spectra predicted by SimLiT and PINO do not match each other making it impossible to select the correct code. We, therefore, decided to study the ${ }^{7} \mathrm{Li}\left(\mathrm{p}, \mathrm{n}_{0,1}\right)^{7} \mathrm{Be}$ neutron source spectra by developing a new deterministic code EPEN - Energy of Proton Energy of Neutron.

\section{Formulation}

The formalism adopted in the present work is very similar to the formalism adopted by Lee and Zhou [3]. However, there are two main differences: (1) The kinematic equations are written in terms of the ${ }^{7} \mathrm{Li}$ mass instead of ${ }^{7} \mathrm{Be}$ mass everywhere due to the fact that the $m_{\mathrm{Li}}$ is more accurately known by an order of two (n.b. at the nonrelativistic limit $m_{p}+m_{\mathrm{Li}}=m_{n}+m_{\mathrm{Be}}$ where $m_{p}$ and $m_{n}$ are the masses of proton and neutron, $m_{\mathrm{Li}}$ is the ground state mass of ${ }^{7} \mathrm{Li}$, and $m_{\mathrm{Be}}$ is the ground or first excitation state mass of ${ }^{7} \mathrm{Be}$ ); (2) The \pm selection criteria adopted by Lee and Zhou in the double-valued region $\left(E_{p} ; 1.92 \mathrm{MeV}\right)$ do not allow us to reproduce the near-threshold neutron spectra presented by them (Figure 4 of [3]) but yields a dip around $30 \mathrm{keV}$. We therefore prescribe our own \pm selection criteria in the double-valued region described below. It is also worth mentioning that our formalism and Ritchie's formalism [4] are very similar except for the definition of " $m_{y}$ " which is introduced as $m_{\mathrm{Li}}$ by Ritchie but considered as $m_{\mathrm{Be}}$ in our approach.

Below the threshold of the three-body break-up reaction, the neutron production is described by the two-body ${ }^{7} \mathrm{Li}\left(\mathrm{p}, \mathrm{n}_{0}\right)^{7} \mathrm{Be}$ and ${ }^{7} \mathrm{Li}\left(\mathrm{p}, \mathrm{n}_{1}\right)^{7} \mathrm{Be}$ kinematics. Any combination of the outgoing angle and energy of a neutron $\left(\theta, E_{n}\right)$ uniquely specifies the incident energy of protons at 
the interaction point $E_{p}=E_{p}\left(\theta, E_{n}\right)$ :

$$
\begin{aligned}
E_{p}= & E_{n}\left(\frac{m_{\mathrm{Li}+\mathrm{p}}}{m_{\mathrm{Li}-\mathrm{n}}}+\frac{2 m_{p} m_{n} \mu^{2}}{m_{\mathrm{Li}-\mathrm{n}}^{2}}\right) \\
& +E_{\mathrm{th}} \frac{m_{\mathrm{Li}}\left(m_{\mathrm{Li}+\mathrm{p}}-m_{n}\right)}{m_{\mathrm{Li}+\mathrm{p}} m_{\mathrm{Li}-\mathrm{n}}} \\
& -\frac{2 \mu}{m_{\mathrm{Li}-\mathrm{n}}^{2}}\left[E_{n}^{2}\left(m_{p} m_{n} m_{\mathrm{Li}+\mathrm{p}} m_{\mathrm{Li}-\mathrm{n}}+m_{p}^{2} m_{n}^{2} \mu^{2}\right)\right. \\
& \left.+\frac{E_{n} E_{\mathrm{th}} m_{p} m_{n} m_{\mathrm{Li}}\left(m_{\mathrm{Li}+\mathrm{p}}-m_{n}\right) m_{\mathrm{Li}-\mathrm{n}}}{m_{\mathrm{Li}+\mathrm{p}}}\right]^{1 / 2},
\end{aligned}
$$

where $m_{p}, m_{n}$ and $m_{\mathrm{Li}}$ are taken from AME2012 [5], $m_{\mathrm{Li}+\mathrm{p}}=m_{\mathrm{Li}}+m_{p}, m_{\mathrm{Li}-\mathrm{n}}=m_{\mathrm{Li}}-m_{n}$, and $\mu=\cos \theta$. $E_{\text {th }}$ is the ${ }^{7} \mathrm{Li}\left(\mathrm{p}, \mathrm{n}_{0,1}\right)^{7} \mathrm{Be}$ reaction threshold energy.

The double-differential neutron yield for one incident proton is

$$
\frac{\mathrm{d}^{2} Y\left(\theta, E_{n}\right)}{\mathrm{d} E_{n} \mathrm{~d} \Omega}=\frac{\mathrm{d} E_{p}}{\mathrm{~d} E_{n}}\left(-\frac{\mathrm{d} x}{\mathrm{~d} E_{p}}\right) \rho \frac{\mathrm{d} \Omega_{\mathrm{cm}}}{\mathrm{d} \Omega} \frac{\mathrm{d} \sigma\left(E_{p}, \theta\right)}{\mathrm{d} \Omega_{\mathrm{cm}}},
$$

where $\rho$ is the volume number density of ${ }^{7} \mathrm{Li}$ nuclei, $x$ is the thickness of the lithium target, $\Omega$ and $\Omega_{\mathrm{cm}}$ are the solid angle of outgoing neutrons in the laboratory and centre-of-mass system, and $\mathrm{d} \sigma\left(E_{p}, \theta\right) / \mathrm{d} \Omega_{\mathrm{cm}}$ is the angular differential cross section of the neutron in the centre-ofmass system. The stopping power $(-1 / \rho)\left(\mathrm{d} E_{p} / \mathrm{d} x\right)$ was calculated by SRIM [6]. By solving the non-relativistic two body kinematic equations, the product of Jacobians becomes

$$
\frac{\mathrm{d} E_{p}}{\mathrm{~d} E_{n}} \frac{\mathrm{d} \Omega_{\mathrm{cm}}}{\mathrm{d} \Omega}= \pm \frac{m_{\mathrm{Li}+\mathrm{p}}^{2}(\mu \pm \xi) \gamma E_{p}}{m_{p} m_{n} E_{p} \xi(\mu \pm \xi) \pm m_{\mathrm{Li}}\left(m_{\mathrm{Li}+\mathrm{p}}-m_{n}\right) E_{\mathrm{th}}},
$$

where the two variables $\xi$ and $\gamma$ are defined by

$$
\gamma=\sqrt{\frac{\xi^{2}=1 / \gamma^{2}-\sin ^{2} \theta,}{m_{\mathrm{Li}}\left(m_{\mathrm{Li}+\mathrm{p}}-m_{n}\right)} \frac{E_{p}}{E_{p}-E_{\mathrm{th}}}}
$$

following earlier works $[3,4,7]$. The \pm sign is corresponding to the two neutron energies for a given $E_{p}$ and $\theta$ in the double-valued region $\left(E_{p} ; 1.92 \mathrm{MeV}\right)$

$$
E_{n}=\frac{m_{p} m_{n}}{m_{\mathrm{Li}+\mathrm{p}}^{2}}(\mu \pm \xi)^{2} E_{p}
$$

where the + or - sign is chosen so that it coincides with the relation between $E_{p}$ and $E_{n}$ in Eq. (1). Above the doublevalued region, + sign is always chosen.

For the ${ }^{7} \mathrm{Li}\left(\mathrm{p}, \mathrm{n}_{0}\right)^{7} \mathrm{Be}$ and ${ }^{7} \mathrm{Li}\left(\mathrm{p}, \mathrm{n}_{1}\right)^{7} \mathrm{Be}$ angular differential cross sections in Eq. (2) at $E_{p} i 1.95 \mathrm{MeV}$, we adopt the $0^{\circ}$ differential cross sections and Legendre coefficients in the centre-of-mass system recommended by Liskien and Paulsen [8]. The ${ }^{7} \mathrm{Li}\left(\mathrm{p}, \mathrm{n}_{0}\right)^{7} \mathrm{Be}$ differential cross section at $1.92 \mathrm{MeV}<E_{p i} 1.95 \mathrm{MeV}$ were obtained by cubic spline fits [3]. Below 1.92 MeV, we adopt the functional form proposed by Macklin and Gibbons [9](See also [10]) assuming isotropic neutron emission:

$$
\frac{\mathrm{d} \sigma}{\mathrm{d} \Omega_{\mathrm{cm}}}=\frac{1}{k^{2}} \frac{g \Gamma_{p} \Gamma_{n}}{4\left(E_{p}-E_{r}\right)^{2}+\Gamma_{\mathrm{tot}}^{2}} \sim \frac{g}{k^{2}} \frac{\Gamma_{n} / \Gamma_{p}}{\left(1+\Gamma_{n} / \Gamma_{p}\right)^{2}}
$$

where $k$ is the proton wave number, $g=5 / 8$ is the statistical factor of the resonance near threshold $\left(E_{x}\left({ }^{8} \mathrm{Be}\right)=18.91 \mathrm{MeV}\right)$ characterized by the parameters $E_{r}, \Gamma_{\text {tot }}, \Gamma_{n}$ and $\Gamma_{p}$. The proton wave number $k$ is related with the proton energy by $1 / k^{2}=\hbar^{2} m_{\mathrm{Li}+\mathrm{p}}^{2} / 2 m_{p} m_{\mathrm{Li}}^{2} E_{p}$. The $E_{p}$ dependence of the width ratio is expressed by

$$
\Gamma_{n}\left(E_{p}\right) / \Gamma_{p}\left(E_{p}\right)=C \sqrt{1-E_{\mathrm{th}} / E_{p}}
$$

with $C=6$ [3].

In order to calculate the neutron source spectrum for a proton extracted from the accelerator at an energy $E_{p 0}$ ("initial proton energy"), lithium target thickness $t$ and geometry of the neutron activation sample (shape, size and distance from the lithium target $d$ ), we prepare tables of $E_{p}$ and $\mathrm{d}^{2} Y\left(\theta, E_{n}\right) / \mathrm{d} E_{n} \mathrm{~d} \Omega$ at various $E_{n}$ (in $1 \mathrm{keV}$ step) and $\theta$ (in $1^{\circ}$ step) for $\left(\mathrm{p}, \mathrm{n}_{0}\right)$ and $\left(\mathrm{p}, \mathrm{n}_{1}\right)$ neutrons according to Eq. (2), and integrate the double-differential yields over the angular range determined by the proton range and neutron activation sample geometry:

$$
\frac{\mathrm{d} Y\left(E_{n}\right)}{\mathrm{d} E_{n}}=\int \mathrm{d} \Omega \frac{\mathrm{d}^{2} Y\left(\theta, E_{n}\right)}{\mathrm{d} E_{n} \mathrm{~d} \Omega} w_{1}(\theta) w_{2}\left(E_{p}\left(\theta, E_{n}\right)\right),
$$

where $w_{1}(\theta)$ and $w_{2}\left(E_{p}\left(\theta, E_{n}\right)\right)$ are the weighting functions controlling the angular integration range, and they are described below.

\subsection{Weighting functions}

\subsubsection{Weighting function $w_{1}(\theta)$}

For a circular neutron activation sample (radius $R$ ), $w_{1}(\theta)=1$ for $\theta<\tan ^{-1}(R / d)$, otherwise $=0$. For a square neutron activation sample (dimension $L \times L$ ), $w_{1}(\theta)=1$ for $\theta<\tan ^{-1}(L / 2 d), \quad=1-(4 / \pi) \cos ^{-1}$ $(L / 2 d \tan \theta)$ for $\tan ^{-1}(L / 2 d)<\theta<\tan ^{-1}(L / \sqrt{2} d)$, otherwise $=0$.

\subsubsection{Weighting function $w_{2}\left(E_{p}\left(\theta, E_{n}\right)\right)$}

When the energy spread of protons extracted from the accelerator is negligible, $w_{2}\left(E_{p}\left(\theta, E_{n}\right)\right)=1$ for $E_{p x}<$ $E_{p}<E_{p 0}$, otherwise $=0$, where $E_{p x}$ is the energy of a proton leaving the lithium target ("exit proton energy"). When the proton stops or slows down beyond the reaction threshold inside the lithium target, $E_{p x}=E_{\mathrm{th}}$. Otherwise EPEN determines $E_{p x}$ by solving the equation $t=$ $R\left(E_{p 0}\right)-R\left(E_{p x}\right)$, where $R$ is the proton range and we calculate it by SRIM [6].

This weighting function should be modified when the proton energy spread is not negligible. If the initial proton energy $E_{p 0}$ normally distributes with its mean value $\left\langle E_{p 0}\right\rangle$ and standard deviation $\Delta E_{p 0}$ according to

$$
p\left(E_{p 0}\right)=\frac{1}{\sqrt{2 \pi \Delta E_{p 0}^{2}}} \exp \left[-\frac{\left(E_{p 0}-\left\langle E_{p 0}\right\rangle\right)^{2}}{2 \Delta E_{p 0}^{2}}\right],
$$

the probability to find a proton with its initial energy $E_{p}$ in the lithium target is

$$
\begin{aligned}
w_{2}\left(E_{p}\left(\theta, E_{n}\right)\right) & =\int_{E_{p}}^{+\infty} \mathrm{d} E_{p 0} p\left(E_{p 0}\right) \\
& =\frac{1}{2}\left[1-\operatorname{erf} \frac{E_{p}-\left\langle E_{p 0}\right\rangle}{\sqrt{2 \Delta E_{p}^{2}}}\right] .
\end{aligned}
$$




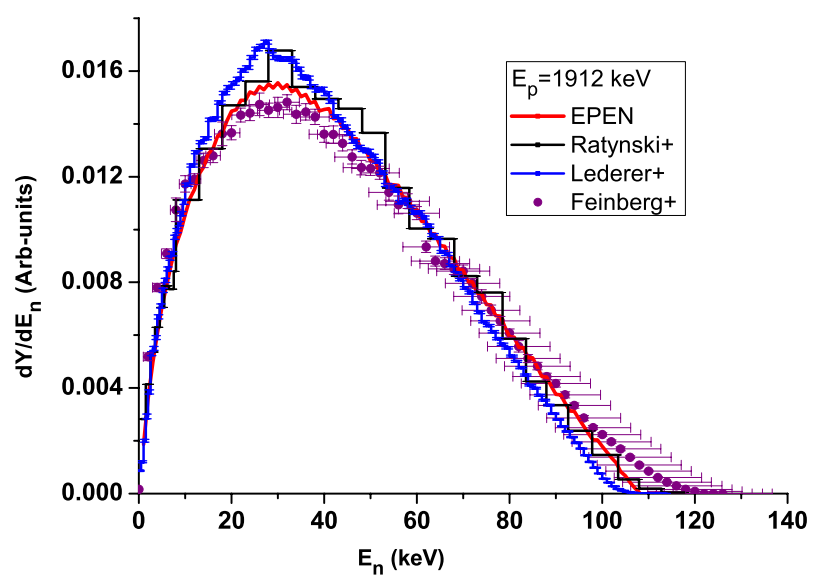

Figure 1. Comparison of EPEN ${ }^{7} \operatorname{Li}\left(\mathrm{p}, \mathrm{n}_{0}\right){ }^{7} \mathrm{Be}$ neutron spectrum at $E_{p}=1912 \mathrm{keV}$ with experimental spectra [11-13] for a thick natural lithium target. The maximum angular range covered is $63^{\circ}$.

In its numerical implementation, we set $w_{2}\left(E_{p}\left(\theta, E_{n}\right)\right)=0$ at $E_{p} \geq E_{p 0+}$ and $=1$ at $E_{p}=E_{p 0-}$, where $E_{p 0 \pm}=$ $\left\langle E_{p 0}\right\rangle \pm \Delta E_{p 0}$. For $E_{p}<E_{p 0-}, w_{2}\left(E_{p}\left(\theta, E_{n}\right)\right)$

$=1$ when all protons stop in the lithium target (i.e., $\left.t>R\left(E_{p 0+}\right)\right)$.

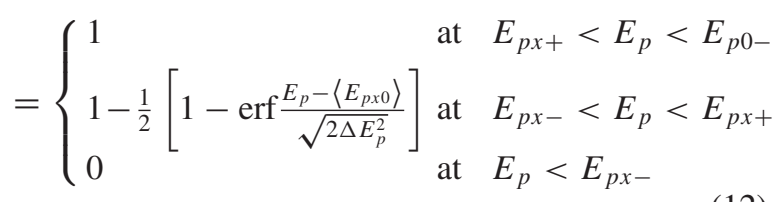

with $\left\langle E_{p x 0}\right\rangle=\left(E_{p x+}+E_{p x-}\right) / 2$ when all protons penetrate the lithium sample (i.e., $t<R\left(E_{p 0-}\right)$.

$$
= \begin{cases}1 & \text { at } E_{p x+}<E_{p}<E_{p 0-} \\ 1-\frac{1}{2}\left[1-\operatorname{erf} \frac{E_{p}-\left\langle E_{p x 0}\right\rangle}{\sqrt{2 \Delta E_{p}^{2}}}\right] \text { at } & E_{p}<E_{p x+}\end{cases}
$$

with $\left\langle E_{p x 0}\right\rangle=\left(E_{\mathrm{th}}+E_{p x+}\right) / 2$ when a part of protons penetrate the lithium target.

Note that the proton exit energy $E_{p x \pm}$ in above equations correspond to the initial energies $E_{p 0 \pm}$ and satisfy $t=R\left(E_{p 0 \pm}\right)-R\left(E_{p x \pm}\right)$, and we assume that $E_{p x}$ also distribute normally.

\section{Results and discussions}

\subsection{Comparison between EPEN and experimental results}

To validate our code, we compare our ${ }^{7} \mathrm{Li}\left(\mathrm{p}, \mathrm{n}_{0}\right){ }^{7} \mathrm{Be}$ neutron spectrum $\left(E_{p}=1912 \mathrm{keV}\right)$ for a $100 \mu \mathrm{m}$-thick lithium target with those measured by Ratynski et al. [11], Lederer et al. [12] and Feinberg et al. [13] in EXFOR [14] in Fig. 1. Our spectrum agrees with the measured ones except for the broader low- and high-energy tails observed by Feinberg et al. [13] which is due to the relatively thick ${ }^{6} \mathrm{Li}$-glass detector and its effects on the time-of-flight resolution according to them.

Figure 2 shows the $0^{\circ}$ double-differential neutron spectra at $E_{p}=1940 \mathrm{keV}$ calculated by us and measured

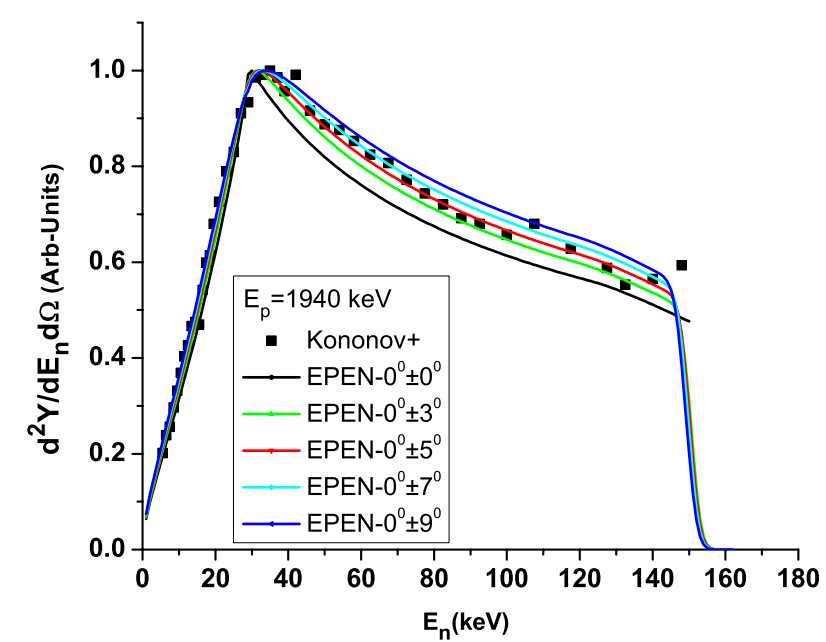

Figure 2. Comparison of EPEN ${ }^{7} \operatorname{Li}\left(\mathrm{p}, \mathrm{n}_{0}\right)^{7} \mathrm{Be}$ double-differential neutron spectrum at $E_{p}=1940 \mathrm{keV}$ for various angular coverage with experimental spectrum [15].

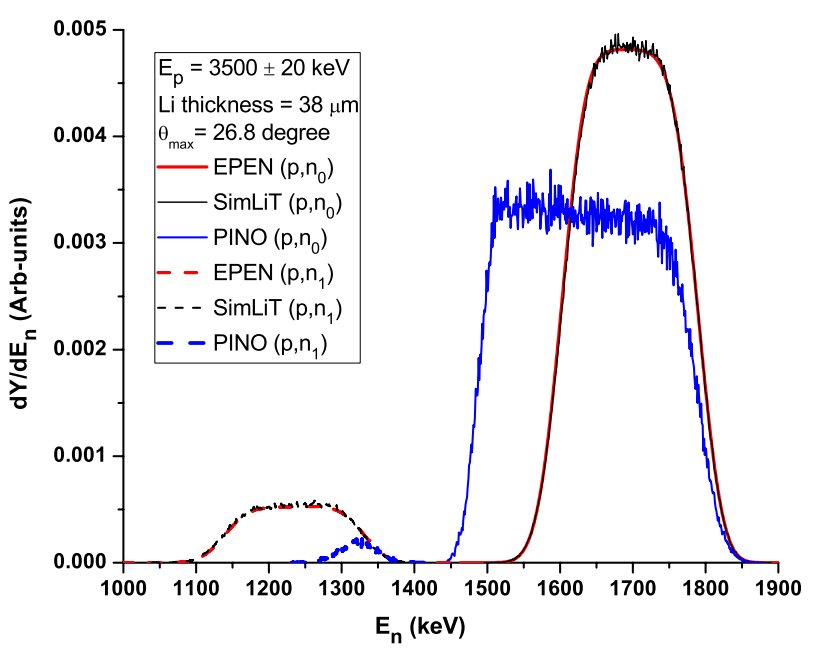

Figure 3. Comparison between EPEN, SimLiT and PINO neutron spectra for a $38 \mu \mathrm{m}$-thick lithium target at $E_{p}=$ $3500 \pm 20 \mathrm{keV}$.

by Kononov et al. [15] taken from EXFOR. It shows a mismatch of the peak position by $5 \mathrm{keV}$ between our spectrum (solid line) and Kononov's spectrum. The angular range covered in the experiment is not known, and we study the angular coverage dependence of the spectrum. The figure shows that the best agreement with the experimental spectrum is achieved when $\pm 5^{\circ}$ is chosen.

\subsection{Comparison of EPEN, SimLiT and PINO spectra}

Figures 3 and 4 show comparison between EPEN, SimLiT and PINO $\left(\mathrm{p}, \mathrm{n}_{0}\right)$ and $\left(\mathrm{p}, \mathrm{n}_{1}\right)$ neutron spectra at $E_{p}=3500 \mathrm{keV}$ with target thicknesses $38 \mu \mathrm{m}$ (thinner than the proton range) and $60 \mu \mathrm{m}$ (thicker than the proton range), respectively. The initial proton beam energy spread (standard deviation) is set to $\pm 20 \mathrm{keV}$. The configurations used in the three codes are set to the same as much as possible, and for the sake of comparison, all spectra are normalized such that the $\left(\mathrm{p}, \mathrm{n}_{0}\right)+\left(\mathrm{p}, \mathrm{n}_{1}\right)$ total neutron yield is equal to 1 . 


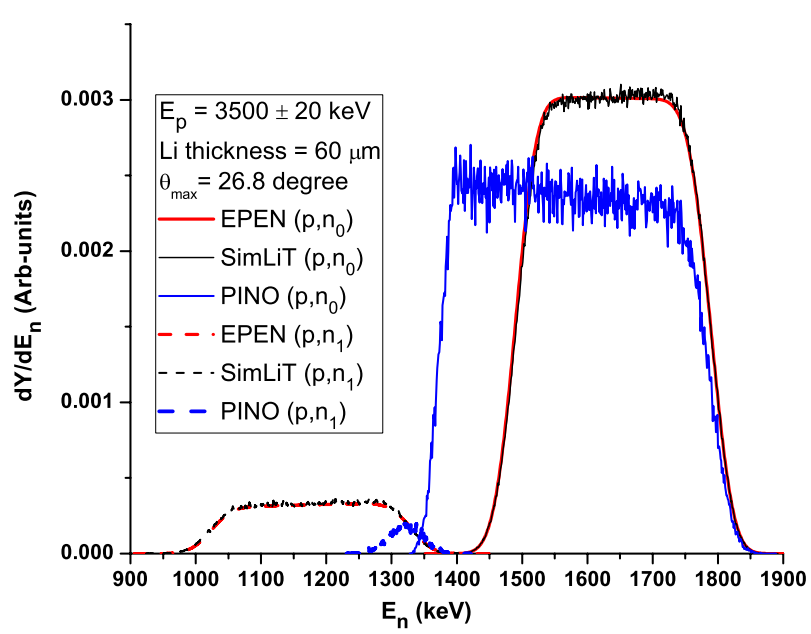

Figure 4. Comparison between EPEN, SimLiT and PINO neutron spectra for a $60 \mu \mathrm{m}$-thick lithium target at $E_{p}=$ $3500 \pm 20 \mathrm{keV}$.

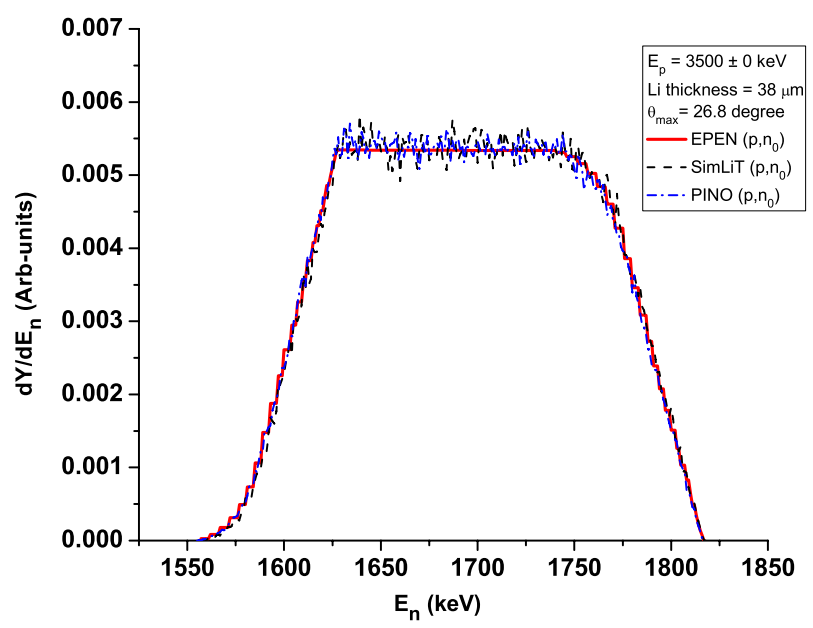

Figure 5. Comparison between EPEN, SimLiT and PINO neutron spectra for a $38 \mu \mathrm{m}$-thick lithium target at $E_{p}=3500 \mathrm{keV}$ without the proton energy beam spread.

Figures 3 and 4 show that there is a perfect match between EPEN and SimLiT neutron spectra for both $\left(\mathrm{p}, \mathrm{n}_{0}\right)$ and $\left(\mathrm{p}, \mathrm{n}_{1}\right)$ contributions while PINO shows large discrepancy from EPEN and SimLiT for both $\left(\mathrm{p}, \mathrm{n}_{0}\right)$ and $\left(\mathrm{p}, \mathrm{n}_{1}\right)$ contributions. PINO shows much narrower $\left(\mathrm{p}, \mathrm{n}_{1}\right)$ neutron spectrum centred near the upper boundary of those predicted by EPEN and SimLiT for both thin and thick lithium targets.

Figure 5 shows that the $\left(\mathrm{p}, \mathrm{n}_{0}\right)$ neutron spectrum predicted by PINO agrees with those predicted by EPEN and SimLiT at the same proton energy when the proton beam energy spread does not exist. This concludes that besides the $\left(\mathrm{p}, \mathrm{n}_{1}\right)$ spectra, the treatment of the proton beam energy spread in PINO is not compatible with those adopted in EPEN and SimLiT.

\section{Conclusion}

We developed a new tool for the ${ }^{7} \operatorname{Li}(\mathrm{p}, \mathrm{n})^{7} \mathrm{Be}$ neutron source spectrum calculation, and studied its prediction against experimental spectra as well as spectra calculated by SimLiT and PINO. A perfect agreement between EPEN and SimLiT was observed for both $\left(\mathrm{p}, \mathrm{n}_{0}\right)$ and $\left(\mathrm{p}, \mathrm{n}_{1}\right)$ neutron spectra at $E_{p}=3500 \mathrm{keV}$. PINO shows much narrower $\left(\mathrm{p}, \mathrm{n}_{1}\right)$ neutron spectrum centred near the upper boundary of the $\left(\mathrm{p}, \mathrm{n}_{1}\right)$ neutron spectra predicted by EPEN and SimLiT. The $\left(\mathrm{p}, \mathrm{n}_{0}\right)$ neutron spectra predicted by PINO agree with those predicted by EPEN and SimLiT only when the proton beam energy spread is negligible. There might be a need to update PINO by improving (1) the treatment of the proton energy spread as well as (2) the proton transport in the description of $\left(\mathrm{p}, \mathrm{n}_{1}\right)$ neutrons.

The present work reveals that the deterministic approach works well for description of "ideal" ${ }^{7} \mathrm{Li}\left(\mathrm{p}, \mathrm{n}_{0}\right)^{7} \mathrm{Be}$ neutron source systems (e.g., pencil proton beam, no surrounding material), and it is usable to study physics of the neutron source system and sensitivity (e.g. impact of the uncertainty in the input cross section on the predicted spectrum). Its output can be used as an input to the Monte Carlo particle transport codes (e.g., MCNP, GEANT, PHITS) to describe more complicated neutron source systems as performed by Friedman et al. [1] by SimLiT+GEANT.

We are thankful to Dr. M. Friedman for providing neutron spectra calculated by SimLiT.

\section{References}

[1] M. Friedman et al., Nucl. Instr. Meth. A 698, 117 (2013)

[2] R. Reifarth et al., Nucl. Instr. Meth. A 608, 139 (2009)

[3] C.L. Lee et al., Nucl. Instr. Meth. B 152, 1 (1999)

[4] A.I.M. Ritchie, J. Phys. D 9, 15 (1976)

[5] M. Wang et al., Chin. Phys. C 36, 1603 (2012)

[6] J.F. Ziegler et al., SRIM - The Stopping and Range of Ions in Matter, SRIM-2013.00 version, 2013

[7] J.P. Theobald et al., Nucl. Instrum. Meth. 95, 1 (1971)

[8] H. Liskien et al., At. Data Nucl. Data Tables 15, 57 (1975)

[9] R.L. Macklin et al., Phys. Rev. 109, 105 (1958)

[10] M.S. Herrera et al., Nucl. Instrum. Meth. B 349, 64 (2015)

[11] W. Ratynski et al., Phys. Rev. C 37, 595 (1988)

[12] C. Lederer et al., Phys. Rev. C 85, 055809 (2012)

[13] G. Feinberg et al., Phys. Rev. C 85, 055810 (2012)

[14] N. Otuka et al., Nucl. Data Sheets 120, 272 (2014)

[15] V.N. Kononov et al., Sov. At. Energy 43, 947 (1977) 